\title{
The multiple faces of social intelligence design
}

\author{
Humberto Cavallin · Renate Fruchter • \\ Toyoaki Nishida
}

Published online: 24 December 2009

(C) Springer-Verlag London Limited 2009

Nowadays, interactions between the human and the digital have become paramount aspects of our everyday experience. The pervasiveness of the digital has closed the bridge between the cyber and the human, progressing toward a situation in which boundaries that used to separate the computers and the world in which the subject interacts with the devices continuously disappear. In a recent conference paper, Mikael and Erik (2008) pointed out how recent trends show how their multiple studies on "digitally enhanced physical environments, as well as by recent research into ubiquitous computing, ambient intelligence, and interactive architecture which all highlight the blend of the physical and virtual world, and the disappearing character of modern information technology", leading them to argue that the "move towards digitally enhanced interactive environments has to be taken seriously by the field of HCI" (Mikael and Erik 2008, p. 21).

The articles included in this special issue of AI and Society, take on the challenge posed by these authors, tackling this subject of study through a conceptual framework that connects the relationship between the individual and the interactive environment by means of social mediation. The theoretical ground to these conceptualizations comes from the paradigm of social intelligence design

\footnotetext{
H. Cavallin $(\bowtie)$

University of Puerto Rico, San Juan, Puerto Rico

e-mail: hcavallin@uprrp.edu

R. Fruchter

Stanford University, Stanford, CA, USA

e-mail: fruchter@stanford.edu

T. Nishida

Kyoto University, Kyoto, Japan

e-mail: nishida@i.kyoto-u.ac.jp
}

(SID) as defined by Nishida that it is explained by Nijholt et al. as "the necessary ability for people to relate to understand and interact effectively with others" and their particular concern to understand "how Social Intelligence is mediated through the use of emerging information and communication technologies" $(2009$, p. 2$)$. This paradigm therefore involves the development of systematic approaches concerning design and implementation of systems and environments, ranging from team-based collaboration systems that facilitate common ground building, goaloriented interactions among participants, to communitycentered systems that support large scale online dialog and instructional design.

The multidisciplinary articles included in this special issue are augmented versions of ten selected works originally presented at the 7th Social Intelligence Design (SID) workshop held at the School of Architecture of the University of Puerto Rico, San Juan, PR, in December 2008. As in previous SID workshops, Puerto Rico hosted for 3 days a multidisciplinary group of individuals, coming from varied disciplines such as industrial design, engineering fields, computer science, human computer interaction, communication, social science, cognitive science, social psychology, and architecture. Common to all, these diverse minds were a shared goal to improve the means and approaches to produce better interactions between the human beings and their environments by means of the principles stated by SID.

The first two articles in this special issue deal specifically with the problem of communication. The article by Katai et al. studies the fundamental structures of verbal communication among people by using the conceptual notions of coming from the Leibnizian notions of space and time. In this article, the formalization generated by the models used by Katai et al. shed light on the pieces and 
connections that underlie the verbal structures of communications between human beings. On the other hand of the communication spectrum, the article by Okada et al. deals with the problem of nonverbal human behavior and how to generate algorithms that can enable computers to capture our communication intentions through body gestures. The approach by Okada et al., presents a process in which the machines incrementally learn the meaning of the body gesturing in an unsupervised manner that does not require a pre-determined number and/or type of gestures that the machine is intended to learn.

A different interaction issue discussed in the article by Merckel and Nishida addresses the problem of how to incorporate augmented reality in everyday devices in order to produce more intuitive ways for human subjects to interact with them. A prominent aspect of their approach, as described in the article, is the way in which the knowledge representation is independent of the interfaces, becoming a multi-device strategy that can be separately managed.

Six articles in this special edition address the interaction between users and technology through three different processes that somehow seem to intertwine: awareness, attention, and engagement. Shiose's article discusses the development of a processing system that creates tactile stereographic versions of images, and how these devices are used to develop social interactions that help develop awareness of the sensory differences between individuals. A different take on awareness through technology is presented by Dadlani who discusses the development of the Aurama awareness system, a device that is aimed to be used as an interface to visually convey information about sleeping patterns, weight trends, cognitive abilities, and presence at home, of elderly people to their families.

A different dimension of awareness is the one discussed by Fruchter and Ponti regarding the social dynamics involved in the distribution of attention and the development of communication across global teams. The article deepens the discussion related to the role that technology plays in creating the context for global teamwork interaction. This type of interaction is studied by means of an academic multidisciplinary global teamwork simulation in which students' role plays to simulate the real life interactions required to design a building. A second article in this issue by Fruchter, Bosch, and Ruhomaki deals with the psychosocial aspects of remote and collocated teams and how perceived collocation versus actual geographical distribution in teams affects the way in which they interact.

In the case of the article by Cardenas et al. SID is used as a framework within which a multidisciplinary course is designed in order to sensitize the students to the role that technology can play on defining the users' experience, and on achieving the designers' goal. Using an academic setting, the experience presented in the article by Cardenas et al. describes and analyzes the experience of developing awareness of the interactions between humans and devices through a twofolded approach that capitalizes on the principles of SID. On one hand, students in the course learn about the social implications of the devices regarding their design and implementation. Similar to the article by Fruchter and Ponti, both students and instructors developed an awareness of the process by getting acquainted with real problems and consequences, the technology used to solve them, and the role that social dynamics plays in generating and implementing solutions.

In discussing the role of engagement as a mechanism to sustain the levels of interaction, the article by Rozendaal et al. explores the dimensions of interaction in game playing. They present an experiment through which they establish correlations between engagement and the experiences of control and content richness of a video game. Their experimental results discuss the role that the positioning of the player in a leading situation plays in the sense of control that is achieved, and how engagement is therefore influenced by social dynamics as well as by the physical features of the game.

Finally, both the SID08 workshop and this special number take advantage of the discussion that the article by Green establishes about the important challenges that SID poses for the evaluation of interactive systems in the years to come. Using a discussion on the Social Intelligence for Tele-healthcare (SIFT) project, Green presents a conceptual background to social intelligence in interactive systems, identifying five challenges for the design of socially aware interactions: the support of user-centered design for social intelligence, the development of a metrics for evaluating social intelligence, understanding the effect of social attributes, the definition of ethical considerations for social intelligence, and the development of modalities to establish and maintain social intelligence as a self-sustainable system.

If as Mikale and Erik suggested, the future of our environments lies in a world in which the digitally enhanced interactive settings will be pervasive, then these five challenges posed by Green will play a central role for the discussions related to Social Intelligence Design, establishing a set of enlightening metrics for future Social Intelligence Design Workshops.

The authors would like to thank all the participants and contributors to SID2008 workshop who shared their interest and work related to this topic. The authors would like to express their gratitude to the advisory and program committee, and all others that helped organize the SID2007 workshop. 


\section{References}

Mikael W, Erik S (2008) Environment interaction: character, challenges and implications for design. Paper presented at the proceedings of the 7 th international conference on mobile and ubiquitous multimedia

Nijholt A, Stock O, Nishida T (2009) Social intelligence design in ambient intelligence. AI \& Society 24(1):1-3 